\title{
Granularity in reciprocity
}

\author{
Caroline Nevejan · Frances Brazier
}

Received: 21 April 2011/Accepted: 8 August 2011/Published online: 27 August 2011

(c) The Author(s) 2011. This article is published with open access at Springerlink.com

\begin{abstract}
Witnessing in merging biological, social and algorithmic realities is crucial to trust, as modelled in the YUTPA framework. Being witness and bearing witness is fundamental to human interaction. System participation in human communities of practice challenges the notion of witnessing and therefore the ability to build trust. Nevertheless, through trial and error, people in a variety of practices have found ways to establish the presence and develop trust in merging realities. This paper presents the results of 20 in-depth interviews with professionals from a variety of disciplines and nations. The conclusion of cumulative analysis is that systems do not witness themselves, but their output deeply affects the mental maps that human beings make of each other, the world around them and their own self. Essential qualities human beings seek when being involved with other beings are defined by granularity and reciprocity in the design of time (duration of engagement, synchronizing performance, integrating rhythms and moments to signify), place (body sense, material interaction, emotional space and situated agency), relation (shared meaning, engagement, reputation and use) and action (tuning, reciprocity, negotiation and quality of deeds). By designing granular interaction in 4 dimensions, reciprocity in witnessing obtains significance and the basis for establishing trust in a variety of presences emerges while human agency acquires potential.
\end{abstract}

C. Nevejan $(\bowtie) \cdot$ F. Brazier

Faculty Technology, Policy and Management, Delft Technical

University, Jaffalaan 5, 2628 BX Delft, Netherlands

e-mail: C.I.M.Nevejan@tudelft.nl

F. Brazier

e-mail: F.M.Brazier@tudelft.nl
Keywords Witnessing - Presence - Trust - Technology · Design · Time · Place - Action - Relation · Granularity · Reciprocity $\cdot$ Agency $\cdot$ Mediated presence $\cdot$ Collaboration

\section{Introduction}

The emerging network society is challenging existing social structures. These socials structures have developed over time, over centuries, across the world. Both in personal and professional contexts, day-to-day practices are changing to incorporate the speed and scale of communication that networks facilitate (Castells 1996). Individuals are discovering beneficial ways to use technology, incorporating technology into their lives in many different ways. People are continually shaping their presence in merging realities and finding new ways to establish trust between each other. New social structures are emerging, and personal lives, organizations and business are adapting.

Accepting the hypothesis that human beings are increasing their participation in 'communities of systems and people', the question that guides ongoing research is 'How should systems be designed for human beings to be willing to accept them?' (Brazier and van der Veer 2009). This paper, based on the open exploratory study 'Witnessed Presence and Systems Engineering', focuses on how people integrate technology in their day-to-day lives. Twenty interviews with professionals in different domains shed light on the process of adaptation to technologymediated network environments from the perspective of the individual human being who performs his/her presence and establishes trust or not.

First focus in the interviews was Witnessed Presence, a phenomenon that is fundamental to social structures. It functions as a catalyst for presence and for trust (Nevejan 
2009). Secondly, the focus was YUTPA framework and its 4 dimensions of Time, Place, Action and Relation through which presence and trust configurations emerge, and this inspired the interviews (Nevejan 2007). As a result, new light is shed on the design of presence and on the design of trust in the current emerging network society.

Section 2 addresses methodology. Section 3 presents theoretical concepts that guide the research: the YUTPA framework and Witnessed Presence. Section 4 focuses on witnessing. Section 5 identifies factors related to the four Yutpa dimensions. Section 6 discusses results and suggestions for future research.

\section{Exploring Witnessed Presence}

The qualitative exploratory study 'Witnessed Presence and Systems Engineering' presented in this paper approaches networked reality from a 'design as research' perspective (Lunenfeld 2003), to understand how people integrate information and communication technologies in their dayto-day practices and the implications of the use of these technologies in their personal lives.

In the context of 'Witnessed Presence and Systems Engineering', 20 in-depth interviews explore how professionals from business, art, design, engineering, journalism, theatre, social science analyse changes in their practice and in everyday life around them. The interviews were held between November 2008 and April 2010. Eleven interviews were conducted with experts in India (8 in Bangalore and 3 in New Delhi), and ten interviews were conducted with experts in Europe (3 in London, 5 in Amsterdam and 1 interview was conducted with an expert from Barcelona). All of the experts have professional careers that are affected by the introduction of technology; their age is in the range of 35-75. The choice of experts is based on diversity of profession, field of expertise, cultural background and their conceptual eloquence.

In the in-depth interviews, the concept of witnessing and presence is explored. Each of the 4 dimensions of the YUTPA framework, Time, Place Action and Relation, is addressed in relation to trust in today's global networked society. The interviews focus on how communication and practice differ 'with the use of technology' and 'without the use of technology'. All interviews are filmed, to trace the lines of reasoning and emergence of new concepts. In the 'thinking interviews', the notions of Witnessed Presence and Trust are the main focus. Principal investigator (Caroline Nevejan) and experts engage in a shared endeavour to question perceptions, to better understand and articulate the phenomena inherent to technology enriched personal communication.
Taking full responsibility for the developing conversation, these interviews can be read as testimonies in which experts bear witness to current ICT's impact on their lives and practices. Experiences, understanding and imagination are explored. As the principal investigator is 'host' to experts' testimonies, the influence of the principal investigator is significant. The constructive process of 'thinking interviews' is the basis for the formulation of a new body of knowledge. In this paper, the experts' contributions are presented in one narrative. No literal citations are provided, but instead, references are made to specific interviews. Transcriptions of all of the interviews, including film fragments, can be accessed at http://www.tmb. systemsdesign.tudelft.nl/witness.

\section{YUTPA framework and Witnessed Presence}

The transcendence of time and place has been a human drive for centuries. Inventions of script, printing, film, radio, television and now the Internet all facilitate the mediation of human presence to other places and other times. These media offer limited perception of the mediated thinking, sounds or visuals. However, when limited sensorial perception is understood in the context of previous experience, human beings have the capacity to construct a sense of presence of the mediated objects and subjects (Tokoro and Steels 2003). While accepting mediated presence as real, human beings learn to assess the variety of mediated presences in their own right. Mediated presence is a trade-off between perception and understanding of the perceived (IJsselsteijn 2004). Human audiences, for example, have learnt that in a theatre, the train on a screen will not hit the first row, even though it seems to come right at them. During a telephone call, people know that the person at the other end of the line is real even though they only perceive sound without additional visual proof. Since the 1990s, the Internet has expanded exponentially and currently social networks facilitate 24/7 connections with family, friends, colleagues and with new contacts. Through steep collaborative learning curves, groups of people assess new possibilities and make trade-off as to how to accept these new contacts/ presences in their communication patterns. Making tradeoffs is not only an individual process, it is also a communal process (IJsselsteijn ibid). Once new technologies are accepted in their own right, they become part of human organizational and business practices. Online transactions have become a multibillion-dollar business within a decade. The speed and scale of the Internet not only changes time and place configurations when mediating presence, but it also offers new possibilities to relate to others and new possibilities to act. 
Natural presence and mediated presence are the result of trade-offs that individual human beings make. Making trade-offs is a collaborative learning process defined by an interaction between cultures and technologies available (IJsselsteijn 2004). From a neurobiological and evolutionary perspective, presence is essentially the strive for wellbeing and survival (Riva et al. 2004). To have presence means to have the ability to steer towards one's own wellbeing and survival. Human beings steer away from pain, from hostility or from danger. Sensations, emotions and feelings are distinct in the performance of presence, and they indicate directions for well-being and survival. Steering towards one's own well-being and survival is the ultimate basis of ethics (Damasio 2004). High trust is much more beneficial for personal lives and organizations than low trust (Fukuyama 1995; Kleiner 2002). Specific configurations of presence design support the strive for wellbeing and survival, while others do not.

In this paper, social structures are described as specific configurations of presence design through which potential trust emerges. Traditionally social structures are based on sharing a unity of time, place and action with others. Today new configurations of this unity are accepted as real communication by millions of people around the globe. The YUTPA framework depicts these new configurations. The YUTPA framework takes 4 dimensions into account, not only time and place but also the possibility to act and the way human beings relate to each other. These 4 dimensions define how people make trade-offs and perform presence themselves to establish trust in the current network society. YUTPA is the acronym for "being with You in Unity of Time, Place and Action'. When any one of the 4 dimensions decreases through the use of technology, human beings adapt by filling the gap by processes of attribution and by focusing on intensity in other dimensions. In the trade-off between the 4 dimensions, not only presence is shaped, but also the conditions for potential trust are created. Different presence configurations offer different possibilities for trust to emerge (Nevejan 2009) (Fig. 1).

Seeking well-being and survival, human beings in technological environments are 'thinking actors': they adapt to their changing environment. Through processes of trial and error in a continuous confrontation between intention and realization, people integrate technology in day-to-day practices, implicitly or explicitly determining trust trade-offs. As 'thinking actors', human beings may not be aware of the configurations in which they partake; yet, by participating, human beings help to produce and support values systems embody (Nevejan 2007). From a design perspective, it is possible to analyse and design specific values in specific contexts (Lunenfeld 2003). From the perspective of value-sensitive design, any system communicates specific values anyway (van der Hoven

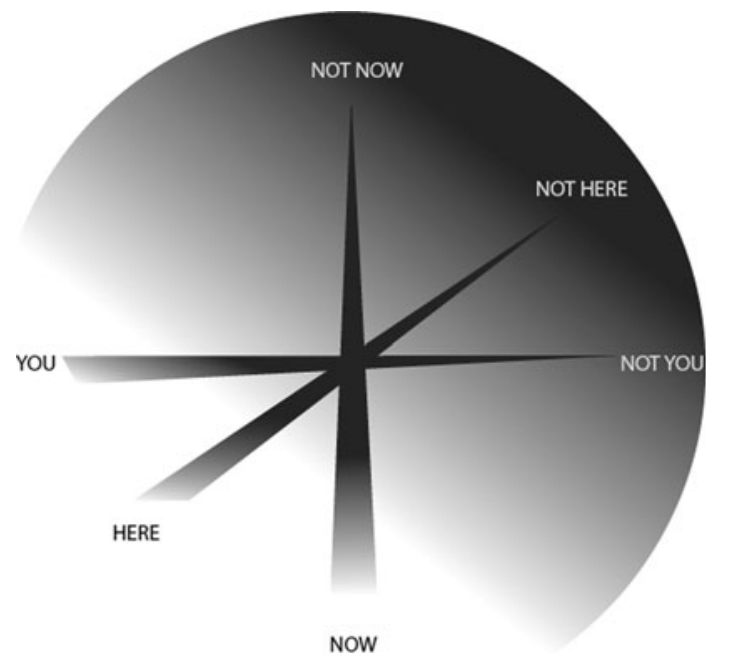

Fig. 1 The YUTPA-sphere depicts the communication spaces of the four dimensions of time, place, action and relation through which specific presence and trust configurations are constructed. The dark and light side of the sphere refer to the possibility to act (graph: Max Bruinsma)

2005). Networking, network, networked and networkmaking power affect social structures because thousands or millions of people participate; yet, few people realize the power structures they are part of (Castells 2009). The YUTPA framework sheds light on how human agency in the new space and time configurations of the network society is constructed (Giddens 1984).

Recognizing each other's spatiotemporal trajectories seems to be a requirement for the emergence of shared concepts and language (Kuhn 2000). In the enactment of being, in the performance of presence, other beings and social structures are essential. Both in natural presence and in mediated presence, Witnessed Presence is a catalyst. An action that is witnessed becomes a deed. A witness has the potential to interfere with a situation to which she is a witness by acting upon this situation and/or by giving testimony about what happened in the situation. Both being witness and bearing witness include the possibility to influence what happens next. A witness accepts responsibility for what she witnesses. Witnessing is key in the design of presence and is key to the design of trust (Nevejan 2009). The capacity to be a witness and bear witness to other beings is the essence of humanness (Oliver 2001). Technology challenges the way people are witness to each other; it challenges both presence and trust designs to the core because scale and speed of communication and transactions is beyond what was ever possible before. Ongoing research into "communities of systems and people' shows that witnessing is a fundamental dynamic in communities of practice (Nevejan and Brazier 2010).

This paper explores how people are witness to each other when using technology and make trade-offs in the 
presences they perform (and accept presences of others) to decide the trust they engage with. Results show that granularity of reciprocity in specific configurations of the dimensions benefits potential trust to build.

\section{Witnessed Presence in communities of systems and people: trade-offs are changing}

This section addresses the phenomena that affect witnessing in on- and offline realities. At the risk of making sweeping generalizations or stating the obvious, the current state of affairs is sketched. Section 4.1 addresses the participation of systems in communities of people. It explores how this participation is blurred, while creating new sensitivities at the same time. It also raises the issue of systems legal position as witness in communities of practice. Section 4.2 sheds light on how people witness, judge and adapt to the global communication arena. Section 4.3 concludes that merging realities challenge embodied knowledge and authenticity, as they were part of human communities until only recent times.

\subsection{Witnessing of systems: systems do not witness people, people witness systems}

For most people, there is a great discrepancy between perceiving and understanding computers. Many people look at a computer, but do not really see nor understand what they are dealing with. When people tune their presence to systems, they train themselves to communicate in the way systems require. Often people will blindly do what the computer tells them to do and suspend their own judgment. A classic example is people who blindly follow their GPS units to narrow footpaths because the GPS unit said to go there. In crisis situations, trust in systems only ameliorates and chances for proper judgment diminish as is shown by research into crisis management (interview Quillinan 2009).

When designing systems, the mapping between human values (what behaviour would they like) and technical values (what are the technical constraints) is very complex. Dynamics of self-organization and adaptation deeply influence how technology is used. However, systems remain dependent on hardware infrastructure, which is located in specific places that are subjected to specific systems of law. In the global network environment, surpassing national boundaries, clashes happen on issues of privacy, data retention, control and monitoring. Values like autonomy, transparency, traceability, security and privacy define the design and social impact of distributed systems. Values of systems are understood locally and are defined by the political and economical cultures within which they function (interview van Splunter 2009).
In European Systems of Law, technological systems are not accepted as witnesses in their own right. In systems of law, the trade-off between presence and trust and between witness and truth has taken several centuries to develop. A witness is a formal position in the process of law for which one has to accept responsibility. Accepting to be a witness is a conscious choice to want to be present and testify. In Court, a witness has to be sworn in by taking an oath. Physical presence is required to be able to judge the witness contribution to the search of the truth. If a witness is elsewhere, the witness has to go to a Court, where he/she is located, to be sworn in by a local judge. Only then can he/ she testify by the way of video or phone connection to a Dutch Court. In this sense, trust is a transferable property between trusted places. Technological obtained proof (like footage of surveillance camera's or DNA reports or twitter logs) is only accepted when experts, who are physically present, testify about their understanding this proof (interview van der Vlies 2008).

From a Computer Science perspective, systems are not participants, nor actors in communities of people (interview Warnier 2009). Systems observe, they monitor, but they do not witness. Systems cannot witness anything on their own because witnessing implies a form of consciousness that systems do not have. However, systems give people context and structure and therefore influence the way people perceive and understand their environment. Here, systems are becoming more powerful over time. Data become more accurate and more interconnected and generate perspectives and representations as never before (interviews Warnier 2009, Quilinan 2009, van Splunter 2009).

When looking at systems, engagement brings the act of witnessing to another level in which larger structure of data and patterns emerge. In most situations, participants witness each other. Algorithmic reality provides the ability to take a new witness position, a 'third point': to construct a global view of a system, its participants and oneself. One can watch the system and the participants in the system witnessing each other, introducing a new kind of reflexivity in which participants witness their own witnessing (interview Hazra 2008).

It is a survival tactic of humans to try to humanize the system and to try to give it more human qualifications. Designers try to make systems more human-like because it makes it easier for humans to interact with systems (interview Warnier 2009). Mathematics has a high aesthetic of its own and because this is translated into rhythms in shape and form, this aesthetic becomes available for people who do not understand the original mathematics at all. Architects are deploying algorithms to create shapes and structures to which people react, sensitive to the aesthetics of this visual logic (interview Jansma 2010). 
Surveillance and identification technologies deeply influence today's society. Large quantities of data are gathered and stored for many different purposes varying from marketing studies to detection of possible criminal behaviour. The nation states now control much more than they did before. In the last century, the state could not control the ability of a citizen to go places to the degree to which they can now (interview MacFaydyen 2009). There are European laws, like the European Data Directive, that define what companies and governments are allowed to store, which kind of information, for how long, and at which point citizens have to be informed. However, these regulations are not very effective (interview Warnier 2009, Quilinan 2009, van Splunter 2009). Interesting in the structure of the witness in judicial European courts is that a witness does not have to incriminate herself, by, for example, remaining silent. In law, presence of a human being (in essence the strive for well-being and survival) is given more agency than the judicial system itself. Consequently, when transposing this concept to systems participation, systems need to be designed to provide human beings the ability to steer towards their own well-being and survival: they do not have to cooperate with processes that may incriminate them. From this perspective, unknown surveillance and monitoring, which may lead to incrimination, is contra-productive and detrimental for human beings. One has to realize that the law took several centuries of evolution and systems participation in human societies is very young and needs to evolve (interview van der Vies 2008).

In conclusion, systems are designed to function in specific ways. Their behaviour is witnessed by human beings who may, or may not, understand what is happening. Systems' capacity to offer larger structures and patterns of data offers new dynamics of reflexivity, which affect human minds and behaviour in new ways that have yet to be explored. Systems participation in human society is very young, and unlike the systems of law for example, fundamental human rights (like the right to not have to incriminate yourself) are not incorporated in the design and organization of systems.

\subsection{Witnessing through systems: transaction, judgment and global adaptation}

When transposing a concept like Witnessed Presence from one culture to another tuning is required. Presence is universal, but the concepts of witness and Witnessed Presence are specific and therefore non-universal. What is considered to be evidence, being witness and bearing witness differ across cultures. An early technology like the fingerprint does not distinguish between cultural identities of people. But when two people meet, the context of the occasion, be it formal or ritual or just in the street, will deeply influence how one person witnesses another. People read the cues in each others' appearances-styles, heritage, cues of community traditions, professional attributes-and this results in a judgment on the other person's identity, be it falsely informed and/or based on prejudice or not. Witnessing is specific to the witness (interview Parthasarathi 2008).

Cultural and political structures of governance define perception and therefore deeply influence testimonies people give. For example, the journalists who covered the Mumbai attacks in 2008 mostly came from well-to-do families who frequented the hotels that were bombed and hardly ever entered the train station that was bombed as well. Even though many more people died in the train station, far more reports on television were about the hotels. Only when cues are equally and fully understood can there be a space for dialogue and transaction. This raises the question whether one can be a witness without dialogue and transaction. Dialogue and transaction is fundamental to Witnessed Presence (interview Parthasarathi 2008). ${ }^{1}$

Processes of judging and witnessing are often intertwined. As one human being witnesses another human being, she also judges the other human being. This is even more so when religion has a strong impact on social and public life. Being an independent woman in Kashmir, Bawa found that judging and witnessing seem to be parallel processes. Only through conversations in which trust grows between human beings, judging may turn into understanding (interview Bawa 2008).

In the global atmosphere, upon which many people are dependent now, this process of judging happens all the time because people are thrown into cultures they do not know. Part of cross-cultural communication is perception of how one person perceives another person, and part of crosscultural communication is being able to reflect on one's own culture. Easily bias comes into play, and one has to balance personal cultural needs, social demands and what is globally required in a professional atmosphere. Also, most international collaborations are dependent on technology and people have to work twice as hard to keep establishing the connection and 'rapport' and develop trust between each other (interview Kaul 2008).

To establish trust in a technology environment is complex. Integrity, sharing information in an honest fashion is a first requirement. Sometimes, there is confidential information that cannot be shared, but at least, it has to be acknowledged. Secondly it is important to show capability

\footnotetext{
${ }^{1}$ Vice versa it can be argued that when transaction is taking place, this can easily be taken for an act of witnessing, fuelling the perception of systems as participants in human ways.
} 
to deliver on time so others see the work has been done consistently and will end up trusting the team. Thirdly, it is important to be clear about intentions and to realize, for example, the purpose of an email and to show that expectation up front. Building reputation is a fourth requirement; one has to realize that trust travels between people (interview Kaul 2008).

Many organizations and business train their employees in global communications skills in which the personal approach is leading to show understanding (instead of just understanding it in your head), to show confidence, to learn to present professionally, to be aware of body language, to develop verbal skills, to manage expectations and to make clear what you need, what you expect, what you want, what you want to do with your team and what you want to do across with the global teams. In the professional global business environment, you cannot be laid back like you are at home; you cannot expect things to happen because the people around you don't know you for 20 years, like your family would (interview Kaul 2008). However, even though people learn to maintain themselves in this highly competitive global business environment, the adaptation to global communication skills has a price.

Adapting to the global communications environment is adapting to a collective imaginary model, which is mostly defined by American industry. It is as if most people are playing a character in a theatre play and dress and eat and relate to others accordingly (interview Upadhya 2008). Drivers of this adaptation processes are higher salaries and potential profit. Working in this global business environment, while at the same time maintaining a local identity in personal lives, people are psychologically torn apart. In the IT industry in India, the effect of this discrepancy between professional and personal identity has led to new stressrelated diseases, which India has not known before (interview Ilavarasan 2008). The question that poses itself is whether and how through global systems technology facilitated communication witnessing emerges. International business experience shows that, next to global communication skills, patterns of presence and absence in physical presence are crucial for success. Collaborators tend to meet at the start of projects and when evaluating the project. In both moments, ethical issues (about what is good to do or was done) are at stake. In between, hierarchy and shared production values create smooth collaboration (interviews Upadhya 2008, Wilson 2008).

Human beings adapt to new power and presence configurations, but also in these new configurations 'facts' matter. People need truth to be able to take responsibility for their own choices and behaviour. There is an indiscriminate recall of vast amounts of data. It is nearly impossible to sift through all information. Complex webs of lies grow and are sustained. Personal experience, including all one has read or seen, becomes the vehicle with which people judge the value of information. Journalists finding and analysing facts are fundamental for democracies to function; yet, the task at hand is more and more complex. Nevertheless, people have to develop strategies to deal with the overload of information because truth is the best agency of consciousness, giving people the power to participate in processes of change (interview MacFaydyen 2009).

Ultimately, online witnessing works only if it supports offline-established cultures, loyalties and relationships. This insight contributes to understanding why social networks, which develop through networks of friends and friends of friends, have such an unanticipated success. Both in on- and offline contexts and in the merging of the two, truth finding by establishing facts and analysing these in connection to each other is more and more complicated but remains to be distinct. For individuals, deciding what to accept or not, personal experience is key in these processes.

\subsection{Witnessing in situations: merging realities challenge embodied knowledge and authenticity}

Today human being's experience evolves in a complex combination of biological and social systems, in which algorithmic systems play an important role. Data images of one's body now influence how the body feels and are the result of a multidimensional reality in which social, biological and algorithmic reality all partake. The sense of sleeping, medical data of sleep patterns, and the sense of awakening in a specific social environment all merge. Witnessing raises questions of truth and trust. 'I witnessed it' is a statement that inscribes the body in the process. That is why, a few decades ago, the camera image was seen as having a certain truth, because a person would have had to be physically present - to be a witness - to have been able to film it. This brings up questions around knowledge and the body and how to approach what is understood to be knowledge. The classical hard empirical approach is that knowledge is only true knowledge when it is experienced through sense organs, but in the present context, the relationship with knowledge formations is immensely mediated through multiple layers of intervention in merging realities (interview Hazra 2008).

The technological systems that humans have developed in the last 80,000 years have been made possible by spacetime and the material properties of the chemicals compounds on planet earth. People have interacted with the natural world in various ways. Their bodies have evolved and interacted over time. Designers and engineers deal with systems that exist in space and time, in which their own body also exists. They have intuitions, and there is that kind of inter-subjective empathy that guides their work. 
Unlike in art, where there is a uniqueness to signature and life experience that can be read and understood, sociotechnical system designs are never unique designs. They evolve, slowly or rapidly; infrastructures as well as human beings change and adapt (interview Sood 2008).

Systems consolidate human behaviour and change human behaviour at the same time (interview Abraham 2008). Good systems only emerge from a practice of human reflexivity. For example, Google gives credibility to a page because real people give value to the page. The more links to a page, the more credible its rating. And people can debunk it. A good system mediates human behaviour with its algorithms. Because the machine mediates human behaviour, this affects 'presence' and 'trust' and therefore raises the issue of identity and of authenticity. As a result, in the merging realities, human knowledge systems change (interviews Hazra 2008, Abraham 2008).

Online, there is hegemony of text. Existence on the Internet only occurs in the infrastructure of knowledge. This subverts existing authenticity in society. There are over a hundred versions of the story of Rama as they have been told over the centuries, but today on the Internet, there are only two or three. Also, young people experience 'giving data away' as establishing authenticity online. That is how one exists online, by opening one self up. Older generations do not experience this as such; yet, younger generations do. In the offline world, one can live with different identities in different worlds. In the online world, one has to find one's own lowest common denominator between the varieties in identities one has, to be able to keep as many people happy. Not only cultures, also people loose multiple nuances of their identity online (interview Abraham 2008).

People's perception of themselves is a complex constructed entity. The notion of signature is the true and most effective manifestation of their inner presence. This in itself is problematic and possibly a 'mirage'. The threshold at which a certain entity ceases to be itself is not clearly defined. Hazra emphasizes that a person can have 500 different ways of signing. The notion of the most original authentic signature or presence of a particular person is of little value in current merging realities. The notion of authenticity is changing. Traditionally, an unexplainable tie to the soil of the land was needed to be authentic, giving a true representation of a specific culture. These are the politics of representation. Today, authenticity has to do more with the degree and intensity of engagement than with supposed organic ties and binds. One can be as authentic in Facebook as on a piece of land for 80 years (interview Hazra 2008).

Having witnessed the IT development in Bangalore since the 1950s, Geetha Narayanan argues that it is time to ask how lived practice generates knowledge. This includes determining the role technology can play in evolving knowledge other than by mechanisms created by a perpetuating thing of hits, tags, social media etc. Self-organization, complexity, co-evolution and adaptation have been the dominant paradigm for the last decades The complexity of current networks is unviable providing the ground for mass hysteria. Narayanan juxtaposes this paradigm by proposing to create a better understanding of knowledge systems of lived practice, to focus on simpler networks and more meaningful networks, in smaller area's and at smaller scale (interview Narayanan 2008).

Qualities in online environments are different from the qualities in face-to-face environments. For example, in face-to-face communication, human beings experience spatial dimension from the perspective of physical embodiment. This does not hold in online communication. Witnessed Presence adds to the notion of presence a dimension of interaction between two beings. Interaction is both being perceived, whilst the other perceives. It is reciprocal, witnessing and being witnessed happen at the same time. In the act of witnessing and being witnessed, necessarily a connection is being established that involves the potential and possibility of some kind of synchrony and rhythm. When meeting face-to-face, human beings 'tune' their bodies to each other in a process of entrainment, synchronizing breath, body movements and more. Transactions emerge from rhythm and coordination. Being in flow with others makes, it possible to communicate in faceto-face contexts and it is necessary for tacit knowledge to be shared as well. In online environments, this is not possible because communication is mostly disembodied, and only series of transactions establish connection. Online communication facilitates a lot of information sharing and a lot of communication, and it may trigger feelings and also rhythms emerge. Both in offline and online environments, synchronization of rhythm is essential and a requirement for witnessing to take place. However, the reciprocity of Witnessed Presence in a face-to-face context is from a totally different quality than the reciprocity in online contexts. (interview Gill 2010).

In the experience of people, different realities merge biological, social and technological. The notion of authenticity is acquiring new meaning referring to engagement in the first place. In the new configurations of reality, existing systems of knowledge, including the tacit, are challenged. Diversity of cultural phenomena and diversity of identity are confined by lower bandwidth in the ever-spreading networks and so it seems. Witnessing in online contexts is reduced to a series of transactions, where in natural presence, a careful tuning of bodies in rhythm and synchronization grants a sense of flow that is beneficial to communication. Both in offline and online 
Fig. 2 YUTPA-trade-offs are defined by different factors in each dimension. These factors can have higher intensity or lower intensity and function in relation with the other dimensions. Specific configurations determine tradeoffs for presence and trust (Graph: Chris Vermaas and Chin-Lien Chen)

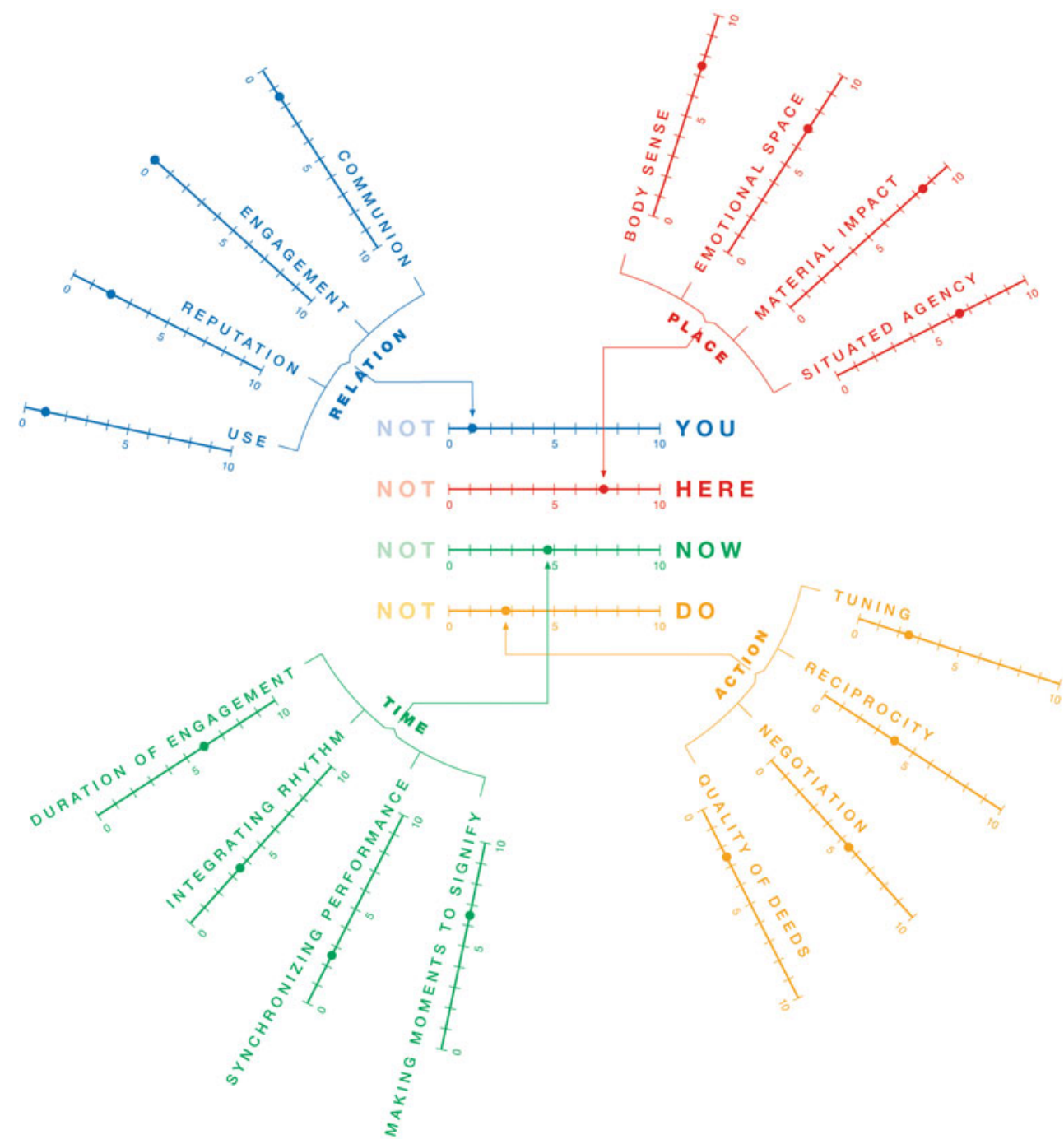

environments, synchronization of rhythm is essential and a requirement for witnessing to take place. However, the reciprocity of Witnessed Presence in a face-to-face context is from a totally different quality than the reciprocity in online contexts. Granularity of reciprocity affects communication and knowledge construction deeply.

\section{Configuring trust through reciprocity and granularity in Witnessed Presence}

In the construction of witnessing as a process of interaction, with or without the use of technology, each of the four dimensions of the YUTPA framework is defined by values of specific factors, which influence the making trade-offs significantly out of which presence and trust emerge. In the interviews with the experts, each of the four dimensionstime, place, action, relation-is addressed specifically in relation to presence and trust. The challenge is to formulate perceptions and understandings that seem to be mundane because technology deeply invades personal lives. As result, in the analysis, in each dimension, factors that affect the trade-off for presence and trust are identified (Fig. 2).

\subsection{Time is beholder of trust}

Where before place was often the beholder of trust, in online communication, time has become the first beholder of trust (interview Hazra 2008). Trust evolves from timing transactions online, whether one is downloading a patch of software, posting on a mailing list or commenting on Facebook (interview Abraham 2008). Online- and systems reality challenges human experience of time. Communicating and collaborating in merging realities needs specific time designs to be satisfactory.

In the establishment of facts and the construction of factual truth, date and time are crucial indicators for connecting evidence with witness reports (interview MacFaydyen 2009). Current concepts of date and time are dependent and tied to place. In this sense, facts are time and place dependent 
Fig. 3 YUTPA factors for time

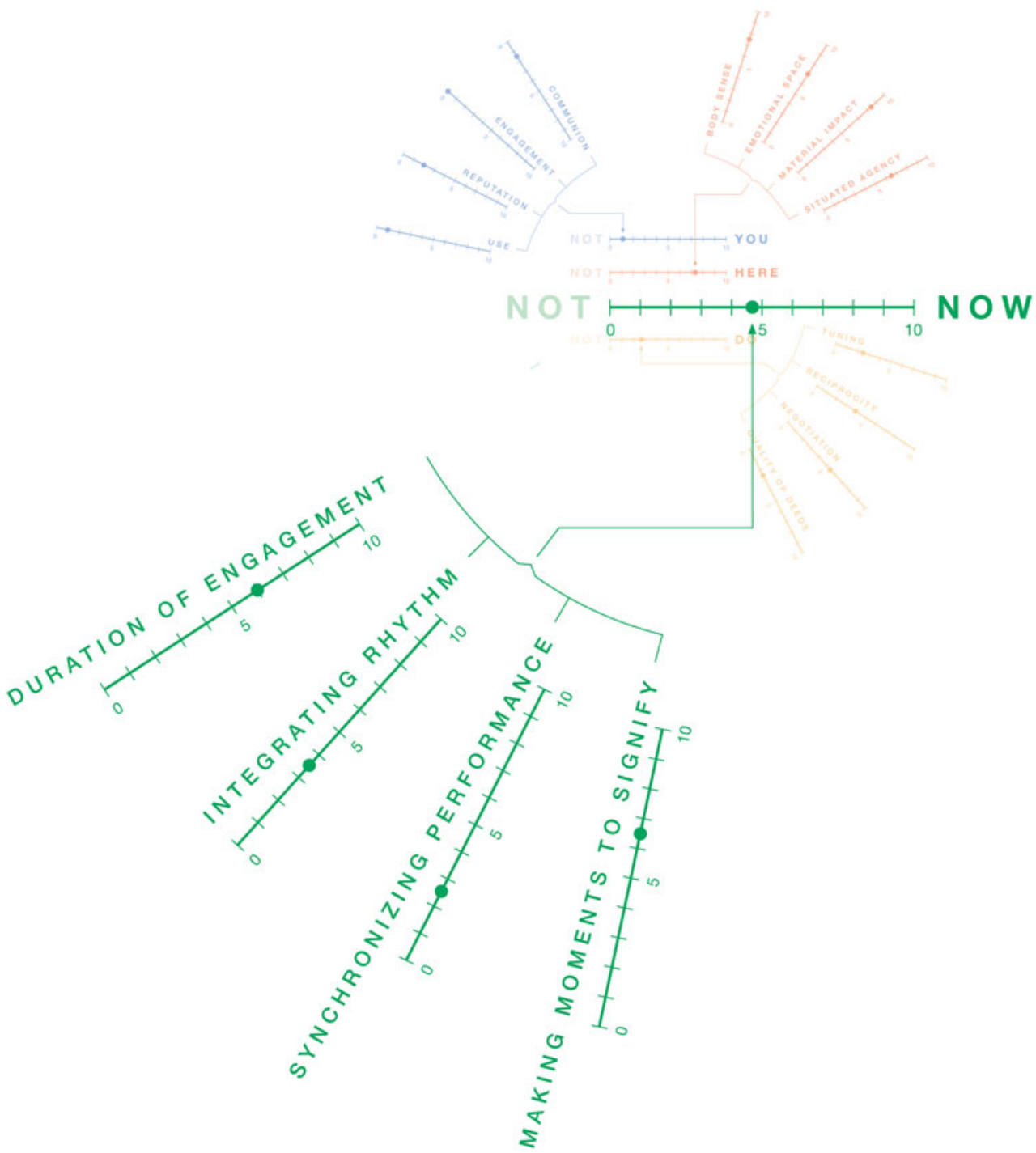

while the experience of the 24/7 information economy seems to offer 'no place and no time' as virtue.

When people do not share place and the specific natureand clock time in that place, a deliberate time design is necessary. In international business, for example, sustainable social online interaction depends upon such time design. When communicating online, people have to integrate their rhythms to each other within the larger organization in which they participate. Work processes are orchestrated in rhythms, and shared rhythm is vital for success. Creation of shared rhythm in online collaboration requires knowing when to meet between time zones, with which medium, for which purpose and for which task. There is no coffee machine where synchronization can take place. The experts interviewed in this study agree that there are specific moments when people need to meet in person to establish trust and truth. Expectation and anticipation are both in on- and offline communications defined by the kind of relationship involved (interview Wilson 2008).
It is crucial to adapt local sense of time to a shared sense of time with clients and collaborators abroad. Regularly, one person is about to go to bed while the other person just woke up; yet, one has to synchronize performance. In the outsourcing industry in India, this has led to completely new social infrastructures where, for example, young women travel by night, restaurants stay open, and family structures adapt (interview Ilavarasan 2008).

When working in the IT industry, unlike many other industries, performance and quality of work can be assessed online. As a side effect, especially in the Global Service Delivery model in India's outsourcing industry (where due to lack of trust between business partners employee's work is logged and monitored $24 \mathrm{~h}$ a day), duration of engagement has become a design issue in itself. Human beings do not appreciate being monitored $24 \mathrm{~h}$ a day; it causes stress and ruptures in identity formation (interview Ilavarasan 2008). People need time off; engagement needs a start and an end for it to be beneficial. The $24 / 7$ economy is detrimental for 
Fig. 4 YUTPA factors for Place

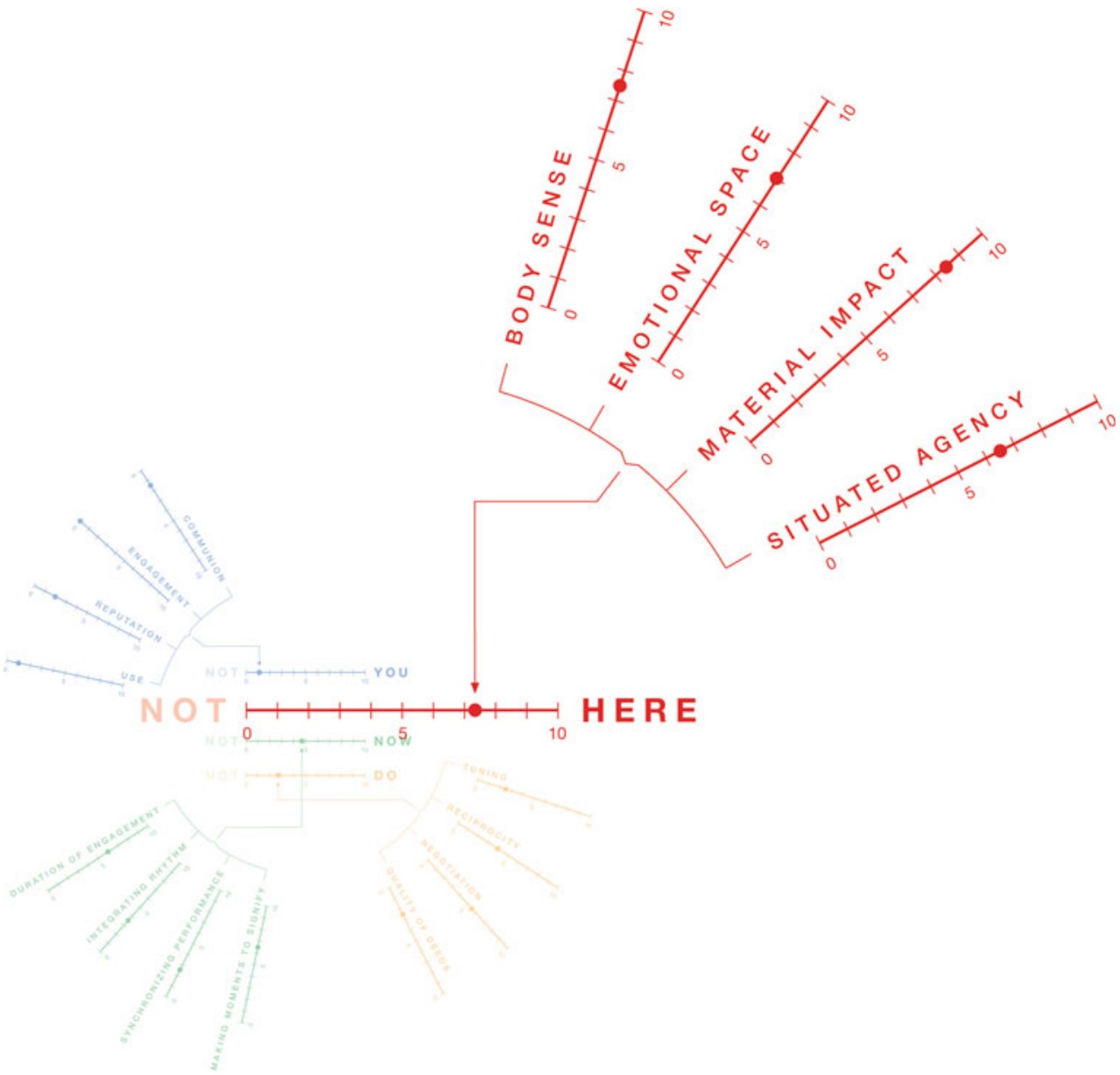

human beings. Systems can be active 24/7; they do not get tired nor get bored as do human beings. Human beings need moments to celebrate, moments of catharsis, moments in which failure or success is shared (interview Narayanan 2008). Failure is fundamental to human growth and takes time to be integrated in a human life, while technology will just treat failure as malfunctioning (interview Narayanan 2008). Human beings need to construct meaning and share this process at distinct moments in time. Systems do not need such moments of catharsis.

When designing time in processes in which systems and people collaborate, differences in scale, organization and experience of time between systems and people have to be taken into account. Duration of engagement, integrating rhythm, synchronizing performance and making moments to signify, are fundamental dynamics from which reciprocity emerges (Fig. 3).

\subsection{Place is defined by engagement}

One of the most remarkable phenomena that emerged from the large-scale use of the Internet is the fact that millions of people are capable of communicating and interacting in vital processes without sharing the place where their bodies reside. The body is present in a place, needs food and sleep, yet people can sense their own and others presence to be elsewhere and communicate anyway. Nevertheless, after a few decades of widespread global communication, it has become clear that locality is of great importance to human beings (interview Upadhya 2008). The same argument is valid for technical infrastructures and the data they transport. Location of infrastructure and location of data define access and flows fundamentally (interview van Splunter 2008). Locality defines situated agency. Situated agency is characterized by cultural and political realities defining both body and data movements around the globe. Politics of presence, including politics of privacy and authorship, are subjected to global business dynamics (interviews Parthasartahi 2008, Dinesh 2008, Warnier 2008).

Place includes social, economical, ecological, social, cultural and historical realities: they are not only material. Sensual perception, but also more complex emotions and feelings, influences the sense of place people have. Emotional space defines the experience of place. The sense of 
Fig. 5 YUTPA factors for Relation

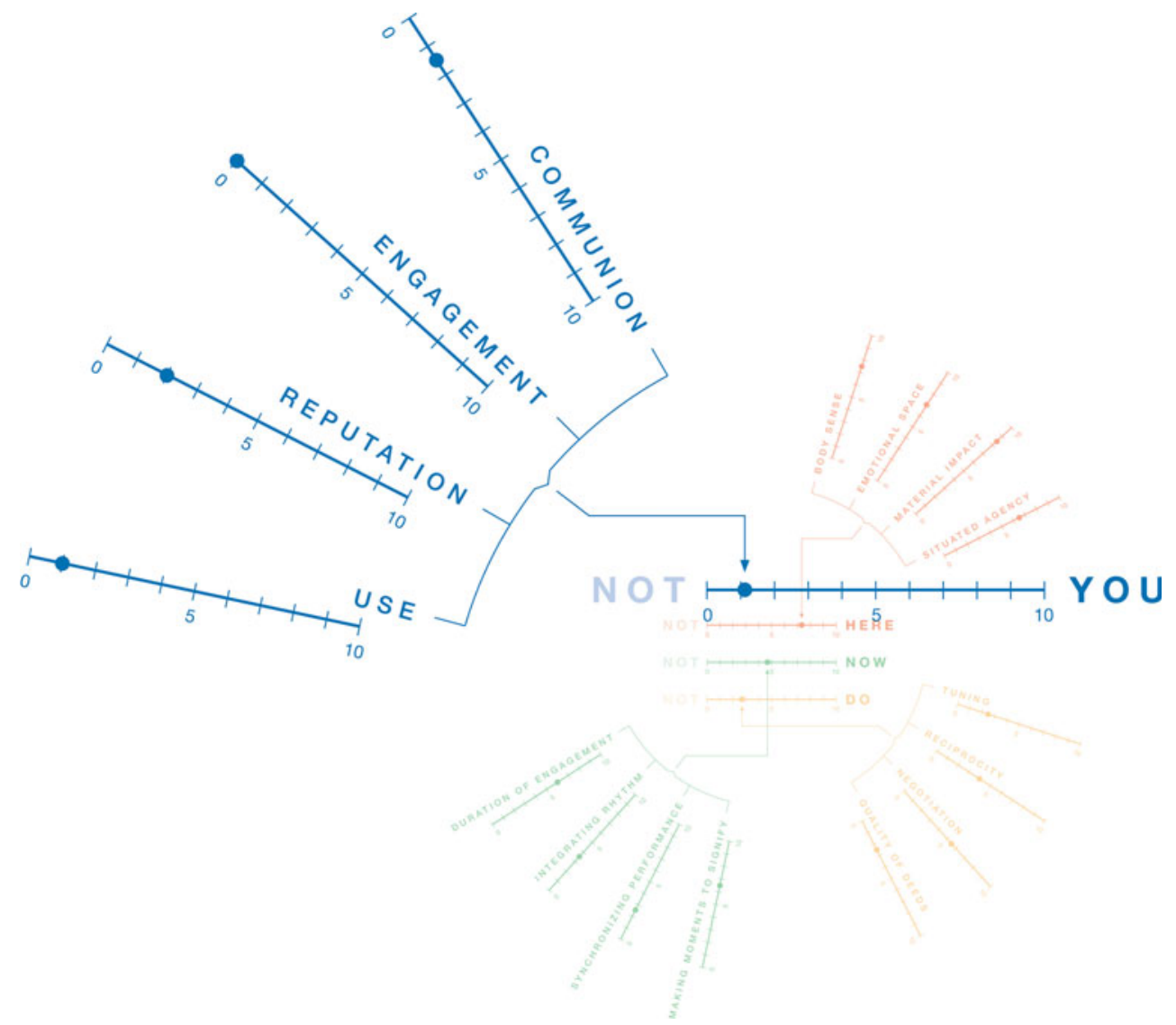

place in any environment is strongly influenced by feelings and emotions. When involved in deep conflict, the sense of space disappears (interview Bawa 2008).

Light is crucial in the experience of place. In theatre and poetry, this is dramatically used. A tragedy starts in a blaze of apparently good light, and then, it goes darker and actually light goes darker. Very often poets, novelists or filmmakers set certain scenes in the rain or in sunlight, because the space one is in replicates what is in the heart (interview Lavery 2010).

The fundamental experience of place is defined by engagement (interview Jansma 2010). Breaking a wall, touching the grass or smelling flowers give physical sensations that create the sense of place. Body and breathing are keys in the experience of real places (interview Jansma 2010). In online communication, the body is also the instrument that records experience (interview Lavery 2010). The sense of place emerges from fundamental reciprocity between body, light, material environment and social feelings. The sharing of sensual reality is for human beings fundamental in connecting to other human beings (interview Gill 2010).

In extreme situations, when being witness in a real place where atrocities occur, the body physically responds beyond a person's own control. Emotions indicate the impact of an event, but they hinder the establishment of facts that are needed to be able to explain what happened, by whom and why. To establish the facts as accurately as possible, in such a situation, a person needs to block emotions if at all possible. An investigative journalist needs to offer facts; other emotional witnesses convey the impact of the event (interview MacFaydyen 2009).

Mediated communication is very effective because representations and shared references do help people to understand what someone else tries to communicate. In the online world, people are mostly dependent on shared references to communicate qualities like taste, colour, smell or touch. References to shared experiences have more impact than a representation, like pictures or video, can have (interview Panghaal 2008). However, people do construct a sense of place, even when information is very limited. Human beings have the tendency to assume they are dealing with a stable universe. This also holds when dealing with mediated communication: people easily attribute missing elements to a situation to create an image of a stable universe (interview Sood 2008).

The sense of place is a sense of orientation and location in the natural world. Points of reference, like a bridge or a tower or a specific tree, make orientation possible. In the online 
Fig. 6 YUTPA factors for Action

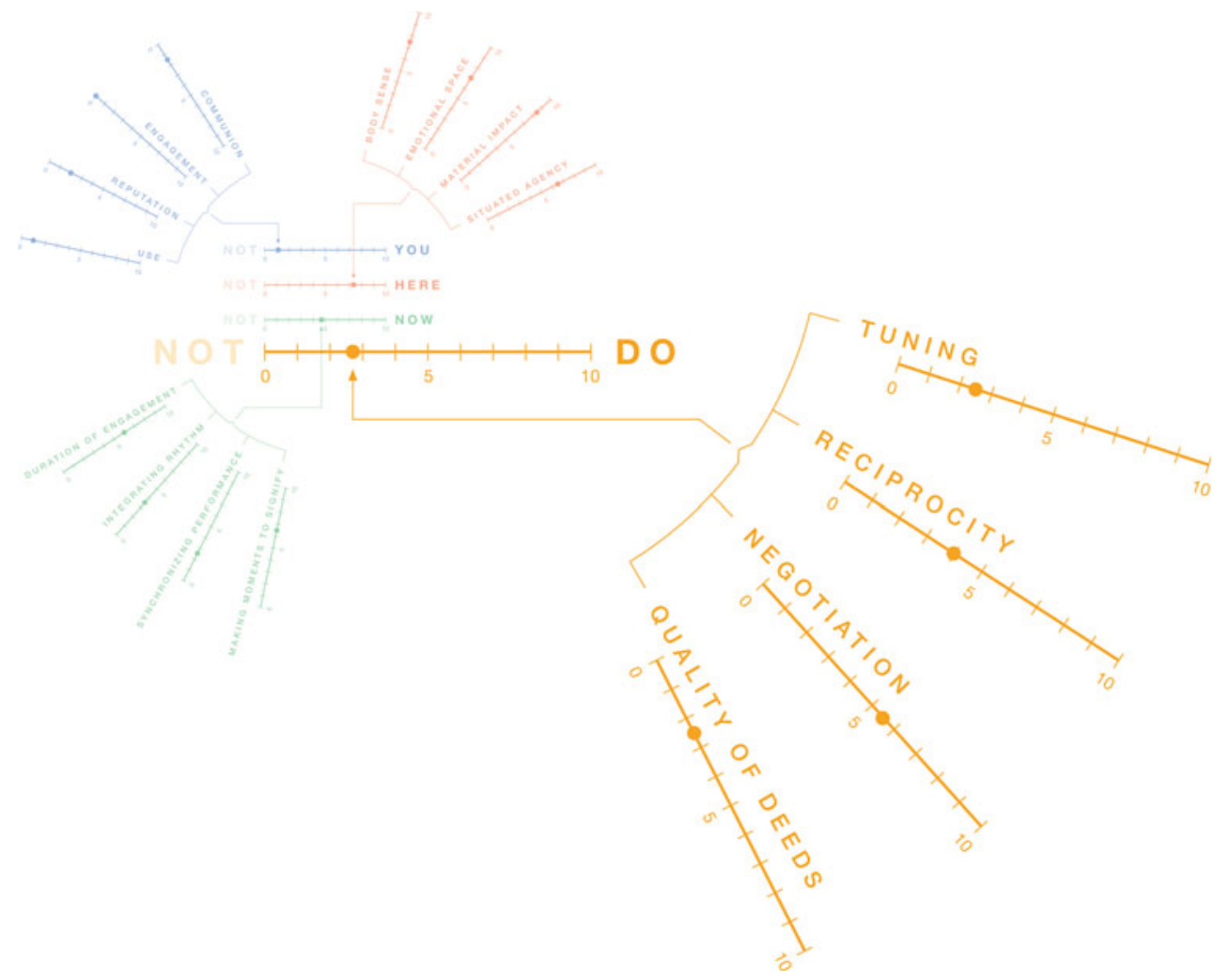

world, neither the sense of orientation nor the sense of location is available. The online world has an algorithmic causality and aesthetics of its own (interview Jansma 2010). In the natural world, it is important for human beings to be centred in the place they are. Their bodies need centredness to be able to endure and sustain in interaction with their material environment to balance body, soul and mind (interview Panghaal 2008). In mediated communication, centredness is not a physical concept. In mediated communication, centre is where 'sense' emerges. 'Sense' can be created in mediated communication (interview van Splunter 2009).

The sense of place is created through engagement with a place, mediated or not, and by accepting the physical sensations and more complex emotions and feelings it holds. The body is the key in the experience of place. By centring one's body, soul and mind acquire the viability to sustain and endure human activity in which the impact of material interaction is distinct. In mediated presence, affecting the human body as well, the centre is where sense emerges. Social, cultural and political realities define activity of both bodies and data in a place significantly. Human beings assume a stable universe and will 'make up' for missing elements, which is also why representations and references to shared experiences are effective. The body as beholder of the sense of place, the sense of wellbeing and centre of engagement, is fundamental to the design of mediated communication. Body sense, material impact emotional space and situated agency contribute to the sense of place in on- and offline environments (Fig. 4).

\subsection{Relation: patterns in granularities of interaction}

In different social sciences, medicine and the humanities, a variety of concepts are used to describe the human being in relation to others and to herself. This section provides insights and formulations of experts interviewed for this study. Four kinds of relations in technology environments are distinguished in these interviews: engagement, reputation, communion and use. Each of these four relations has psychological, sociological, philosophical, medical and cultural consequences. The first insights of the experts are described in their own terms.

The experience of Facebook is difficult to communicate to someone who has never experienced Facebook or a comparable social network. This also holds for professional international collaboration (interview Wilson 2008). In both cases, the dynamics of interaction are defining. In theatre, one of human's oldest mediated formats of presence, there are no relations without actions; only actions build and change relations (interview Lavery 2010). Communication on the Internet is highly text dependent, generating words as activities and 'words that act'.

In natural presence, tuning is a physical interaction testing the boundaries of social contracts. In online 

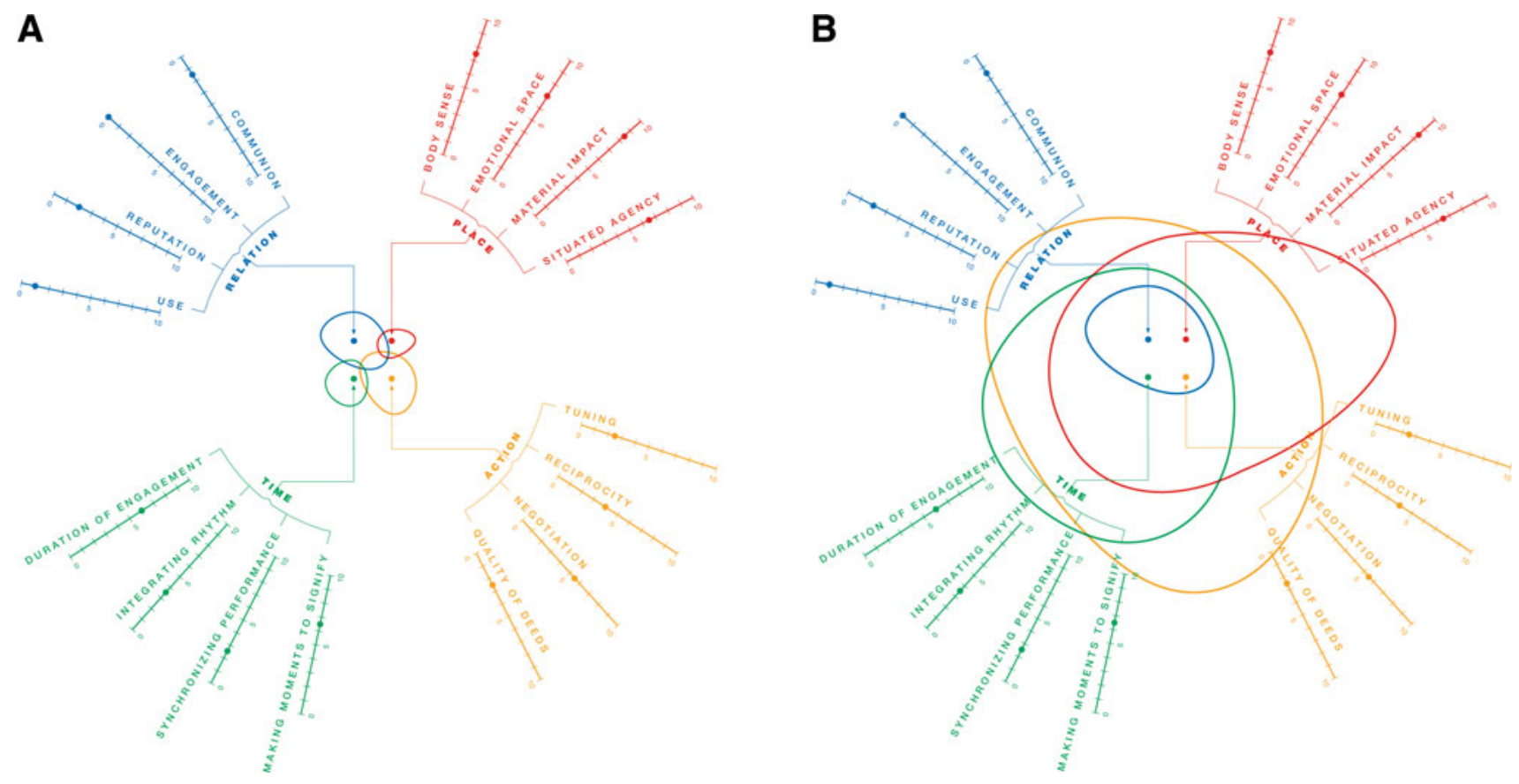

Fig. 7 a, b inspired by Venn diagrams this visualization shows strong and weak areas of trust

environments, tuning is more complex. Complex dynamics characterize such tuning moments. Who is the boss? What is the right tone? How to fit in? And these are also influenced by expectations, which are the result of previous experiences in real and online spaces (interview Abraham 2008). There are no universal rules for handshakes in an online community. The community corrects each participant, through comments and remarks, till everybody is equally uncomfortable (interview Abraham 2008). Global communication skills help people to perform their presence in variety of online environments (interview Kaul 2008). Such skills do not allow for a lot of diversity. Both identities and cultural nuances disappear, as discussed before (interview Abraham 2008).

When discussing communities of people and systems, a distinction has to be made between 'use' and 'being in communion with'. A user is someone who is aware of the instrumentality of his or her actions with objects and systems with which he or she interacts. This is distinct from interactions with other natural and intelligent life forms for which there is communion; there is the possibility of a shared meaning. Understanding perception can be confusing, but people are not confused in their perception of whether they interact with systems they use or beings with whom they are in communion (interview Sood 2008). People make clear distinctions in their vocabulary when they speak about things versus beings. In many online systems, a clear effort is made to blur the distinction between systems and beings, demanding human beings to format their presence to system needs (interview Quillinan 2009). Human beings, however, notice the difference. When designing systems, objects or architectures, the designer communicates a set of values that users may recognize or even sense (interview Jansma 2010). Users may enjoy but are not in communion; there is no generation of a shared meaning (interview Sood 2008). Sharing experience, including establishing a shared meaning, is a fundamental basis for trust between human beings (interview Jansma 2010).

Both in a context of 'use' and in a context of 'of being in communion with', there is the possibility of engagement, referring to a perseverance of attention and interaction that generates its own dynamics. Engagement is intensity of dialogue, sustained interaction, focused and fine grained. Engagement is an attitude in which the sense of self dissolves in the situation in which a person is engaged, whatever the outcome may be (interview Hazra 2008). The distance between self and other disappears. An attitude of engagement results in for example 'if someone feels hunger, I feel hunger' (interview MacFaydyen 2009). In online larger data structures, however, it offers dynamics for reflexivity that are new (interview with Hazra 2008).

A fourth crucial dynamic for relations online is reputation. On the Internet, large groups of people contribute to shared knowledge environments, to social networks, to Open Source software libraries and more. This dynamic is not defined by being in communion with each other to find a shared meaning, but mostly by performance to build 

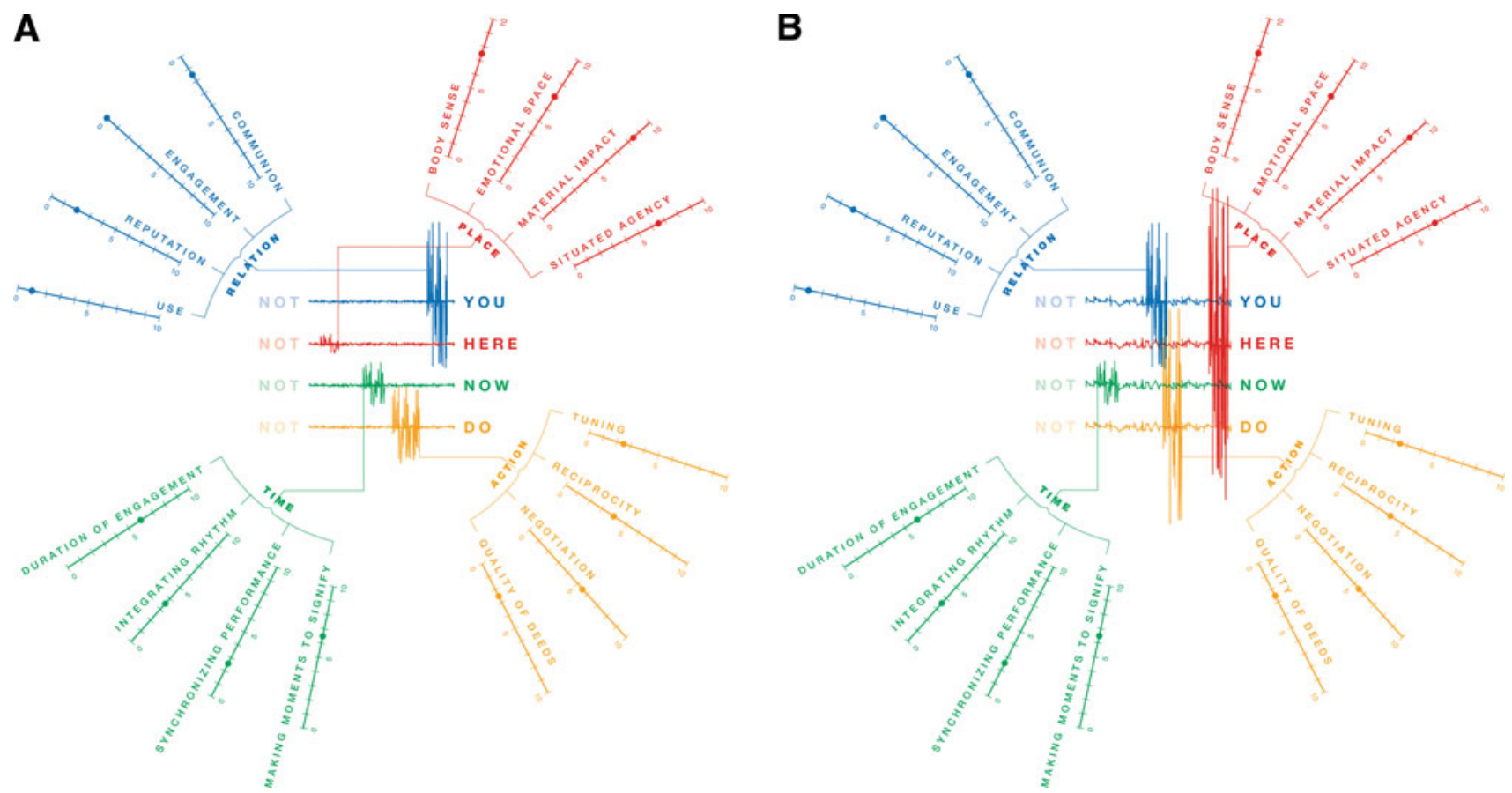

Fig. 8 a, b Trade-offs define the 'tone' of communication, inspired by the French saying 'C'est le ton qui fait la musique

reputation. In, for example, the open source community, releasing software is publicized, and contributors work for an audience. Failed performance towards the deadline of a release date damages a person's reputation and generates loss of credibility. Previous successful contributions to documentation, bug reports, patches or CBS archive, contribute to establishment of reputation. They are acts of performance within the legal framework that defines access and ownership. The hierarchy of trust and authenticity is based on current and earlier performance in the community. Emotions evolve in relation to the performance to be recognized, to fill the space etc. Benevolent dictators of the meritocracy orchestrate and mediate these performances, emotions and relations between the participants in the community (interview Abraham 2008).

There is a great difference in dynamics between relations that are based upon the possibility of a shared meaning, on use, on reputation or on engagement. Each kind of relationship offers different patterns of granularity in interaction with tune presences in distinct manners, which in turn define how trust may emerge (Fig. 5).

\subsection{Action and reciprocity: trust sets the scene}

Action is the final dimension to be discussed in this paper. The interviews showed that action is very tightly linked to reciprocity and trust: the experts could not discuss them separately. Trust sets the scene for possible actions to do.
In theatre, the distinction is made between actions and activities. Actions affect identity and what happens next. Activities, drinking a cup of coffee of doing the dishes, just happen and have no consequence (Interview Lavery 2009). In this paper, the focus is on actions; they are fundamental to the finding of facts (interview MacFaydyen 2009).

Actions in online environment are mostly done through words. To understand how 'words act', a variety of terms is used, and these words are not distinct in meaning: information, communication, tuning, transaction, interaction, negotiation, reciprocity, representation, reference, replay and more.

Human beings need initial trust to be able to act and participate in both their physical environment and virtual and online environments. In face-to-face contexts, transactions emerge from rhythm and coordination (interview Gill 2010). In the first few moments of meeting, someone people have a sense of how to engage and how to trust. This is before any other action takes place. In an online environment, this takes longer. Trust emerges after transactions have taken place, and coordination is established. The establishment of trust in on- and offline contexts has different trajectories. In the real world, synchronization and tuning of rhythm is inside out. In the online world, trust emerges outside in, through series of coordinated transactions (rhythm and synchronization) through which trust develops over time (interview Gill 2010).

In online environments, trust emerges when a series of transactions become interaction, in the transition from 

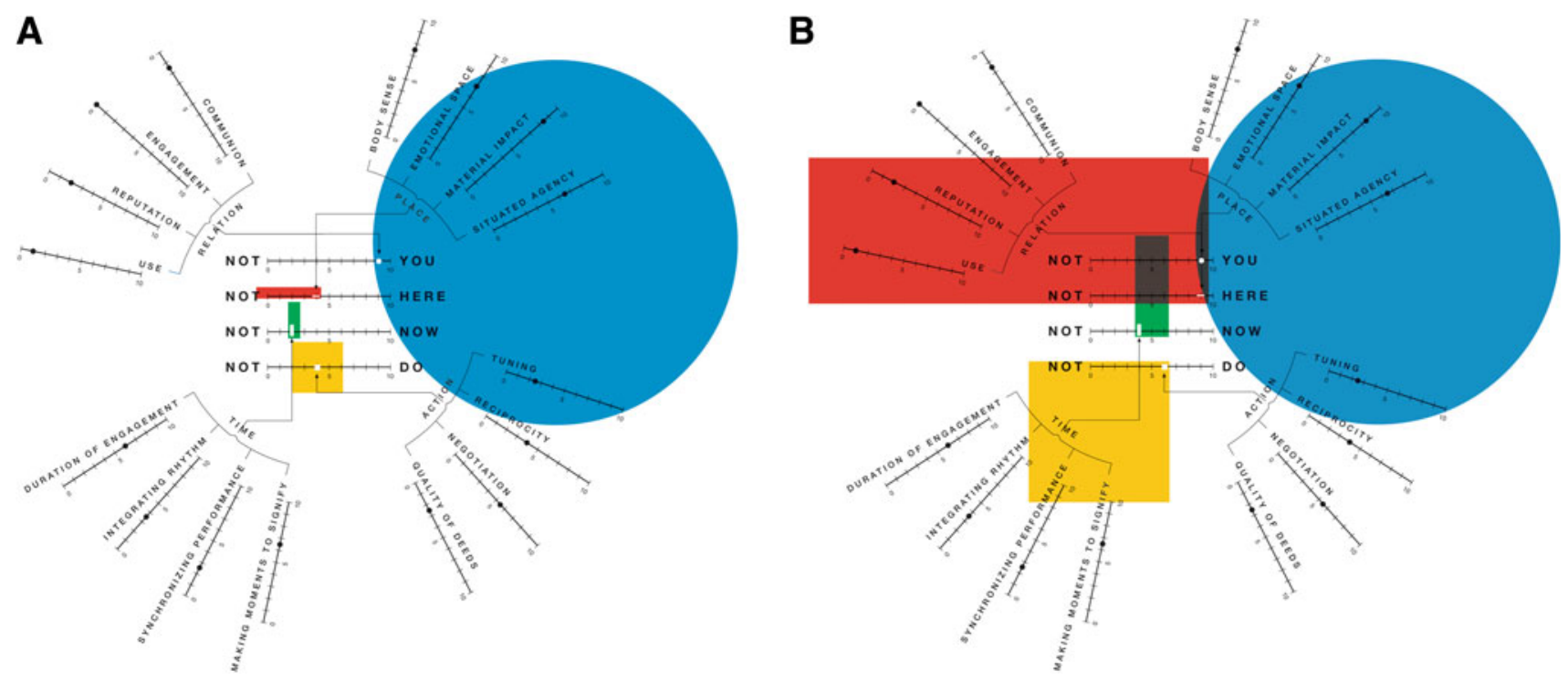

Fig. 9 a-c certain dimensions are 'carrier' of trust in specific configurations and define trade-offs more than others

cognitive understanding to feeling. When random noise turns into rhythm, feeling emerges (interview Gill 2010). Establishing rhythm in online environments requires careful negotiation at first. It can be compared with 'courting' in a sense. In this negotiation, both convention and spontaneity play a role. The online world needs duration of engagement before the advantage of a convention is acquired. Online worlds do not have the spontaneity of a face-to-face dialogue and are more fragmented (interview Gill 2010). The inside-out-trust trajectory, which characterizes trust in face-to-face contexts, can be compared with human beings try to find a common musical composition. The outside-in-trust trajectory, which characterizes trust in online environments, can be compared with the hard negotiation of armies negotiating their terms of openness for survival's sake (interview Gill 2010).

The nature of reciprocity fundamentally changes in mediated presence. When, for example, mediating music, the nature of the interaction between a performer and a listener changes profoundly in mediated formats. The primary quality of the real singer and the real listener is the reciprocity between them. This fundamentally changes when it is mediated. Music can be recorded and be listened to at any time and any place. The relation to the voice of the performer has changed. The voice that could only be heard before at a special occasion is set free from the boundaries of time and place. As a result of this, the interaction between the performer and the listener changes profoundly. The site of performance changes, the relation between the voice and the ear, the relationship between the place from where the voice is coming and the place where the voice is heard change. When replaying the music, the experience has become anonymous both ways: for the listener, because there is a voice but no face, and for the singer, because there is no presence of the audience with whom to interact. Nevertheless, when listening to music that is replayed by a CD for example, the listener attributes different qualities to the music to which she listens. These are not based on a real-life interaction with the singer but are sensorial. The replayed music has the potential to influence the listener: behaviour, mood, sense of calm, imagination and more. The listener creates a mental map of the replayed music, and this is also influenced by other information (interview Parthasarathi 2008).

In the online world, not only music but also human communication is based on mental maps. Human beings have mental images of each other on the basis of which they communicate. Such mental images are dynamic and dependent on processes of attribution. Processes of attribution are triggered by transactions online. Online existence is based on these transactions: people have to continually do transactions with friends, colleagues, and others to prove presence in time and space. Posting on a mailing list and commenting to Facebook, twitter, sending emails etc., are transactions that contribute to the negotiation of interacting identities. Digital witnessing contributes to the establishment of reputation and authenticity, through hyperlinks and records of transactions between humans and machines as well as between humans mediated through machines. When a system comes into being, branding and social context create its credibility. When a system is established, its moments of crises and celebration will move its credibility in one way or other direction (interview Abraham 2008). 


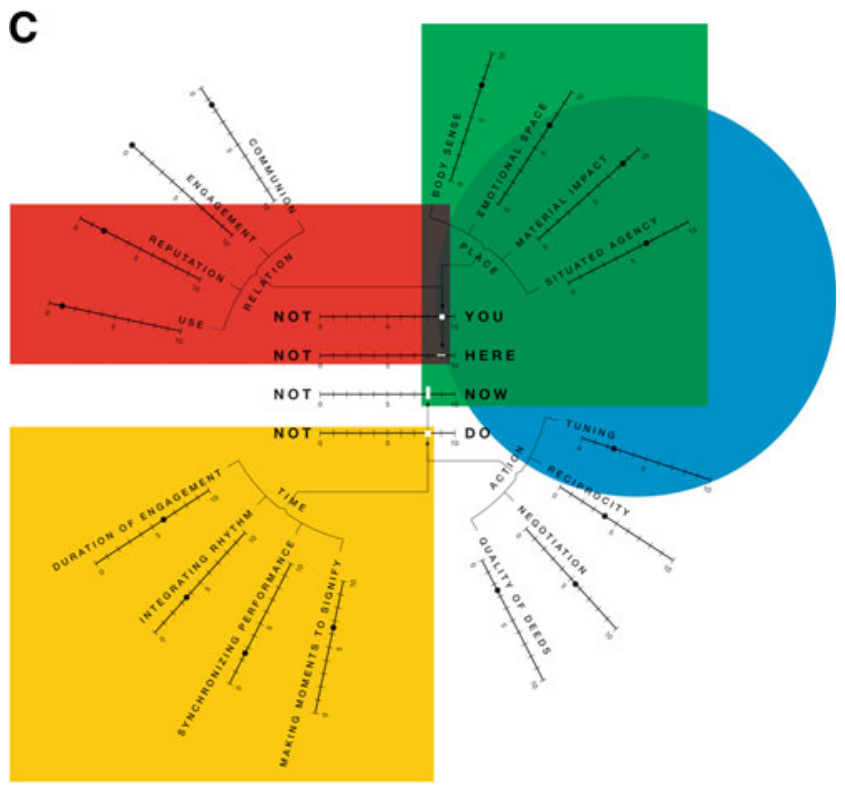

Fig. 9 continued

People manage their identity online, and this is often time consuming. Professional life depends increasingly on keeping virtual identities going. The use of technology is deeply defined by class and culture; the use of technology in the bottom segment of society is critically different from the elite. The elite sector, which is geared towards going up the social ladder, is focused on personal assets: personal memories, life blogs and pictures. People at the bottom of the pyramid, however, are looking for sharing, connecting, collaborating and creating 'oneness to go forward' (interview Narayanan 2008).

Human beings give trust to systems and base their trust on systems as well. Basic trust in a system is required to operate and participate. Negotiation of trust is often needed with many different parties, including software and hardware providers, network providers (interviews van Splunter 2009, Abarham 2008). Governance 'of' communication systems (ownership and regulations, agency of regulation) and governance 'through' communication systems (with information transactions, accountability, transparency, efficiency) both fundamentally define possibilities and consequences of participant's acts online (interview Parthasarathi 2008). Much initial trust in technology is based on ignorance and perception instead on reality and analysis of the implications of its use (interview Abraham 2008). Negotiation between transactions and interactions in online reality has different dynamics than in offline reality. This affects human agency profoundly. Tuning presence for action in online environments is the result of a conscious exchange of transactions. However, failure sometimes occurs. Human beings deal with failure with their hearts and their minds. Technology deals with failure as a statistic. Technology can provide data as input for decisions, but the meaning of data is contextual and technology can only partially know (interview Narayanan 2008).

Information has become more readily available in current networked societies. People are continually informed about the state of the world and may feel witness to what is happening in the world. However, the implications of vast amounts of information for the ability to act are still unknown. (interview Parthasarathi 2008).

This section argues that tuning, reciprocity and negotiation are fundamental to the dimension action on the basis of the interviews. All experts also referred to a fourth factor namely that of quality of deeds using a variety of terms ranging from transaction, interaction, communication actions, activities and performance. Online deeds often affect the physical world, sometimes directly and sometimes indirectly. The distance between cause and effect impacts the character of a deed. That is why 'quality of deeds' is a fourth factor to be taken into account (Fig. 6).

\subsection{Trade-offs are made}

Between the value and nature of factors in each of the four dimensions, trade-offs are made. Trade-offs define how people perform their presence and engage with potential trust. Trade-offs are the result of negotiation between perception, expectation and intention. Together with designers, Chris Vermaas and Chin-Lien Chen visualization of trade-offs has been explored. Results are shown below (Figs. 7, 8, 9).

\section{Conclusion and further research}

The exploratory study Witnessed Presence and Systems Engineering shows that trade-offs in performing presence and deciding to trust are changing because of system participation in human communities of practice. Notions of embodied knowledge and authenticity are challenged. System participation has opened up a new range of possibilities to act and to be witness to each other. Configurations of time, place, action and specific relations in which people engage, define whether and how people trust each other and the structures and systems they are part of. Witnessing is specific to the witness. Dialogue and transaction are fundamental to Witnessed Presence. Reciprocity and granularity in each dimension and between dimensions contribute to building trust.

Further research will study how trade-offs for presence and trust in specific configurations are established. Given the outcome of this study that being witness and bearing witness has acquired new dynamics, future research will explore whether and how specific actions in specific 
relations require specific time and a specific place design, reciprocity and/or granularity.

Secondly, further research explores how the identified dynamics for trust contribute to values of systems design: autonomy, transparency, identify-ability and trace-ability. These values, identified in an interdisciplinary study between Law and Computer Science, focus on human agency in relation to intelligent distributed systems (Brazier et al. 2004).

Future research will focus on the dialogue of the inner witness of human beings and their mental models. Currently, 12 artists are making work to answer the question 'What happens when one witnesses another?' The effect of dramatization and imagination as part of the human being's survival kit, and their effect on trade-offs, is being explored.

Acknowledgments Authors thank the reviewers of this paper for contributing valuable insights, Leen de Baat for filming the interviews, Thomas Quillinan for making the website where source material of this study, both film and text, can be found. This study is generously sponsored by Stichting NL net, VU University Amsterdam and Delft Technical University in the Netherlands.

Open Access This article is distributed under the terms of the Creative Commons Attribution Noncommercial License which permits any noncommercial use, distribution, and reproduction in any medium, provided the original author(s) and source are credited.

\section{Appendix}

Interviews can be found at http://www.systemsdesign. tbm.tudelft.nl/witness (accessed 26 April 2010) For convenience, short descriptions of the interviewed persons are given below.

Sunil Abraham is director, Policy of the Centre for Internet and Society, and is a Bangalore-based social entrepreneur and Free Software advocate. He founded Mahiti in 1998, which employs more than 50 engineers today. Between June 2004 and June 2007, Abraham managed the International Open Source Network, a project of United Nations Development Program's Asia-Pacific Development Information Program, serving 42 countries in the Asia-Pacific region.

Zainab Bawa works as an independent researcher on issues of urbanism, governance and impact of technology on political practices and institutions. Currently Bawa is pursuing her $\mathrm{PhD}$ from the Centre for the Study of Culture and Society (CSCS) in Bangalore. In the past, Bawa has travelled extensively and has worked collaboratively with researchers in Kashmir and in Bangladesh on issues of space, conflict, violence and their impact on society.

Dinesh is computer scientist from Bangalore. He collaborated with Paul Klint at the Centre for Mathematics and Computer Science in Amsterdam for several years. When he returned to India, he started SERVEOTS, a web service provider for small to medium enterprises.

Satinder Gill is based with the Centre for Music and Science, University of Cambridge. She received her $\mathrm{PhD}$ on 'Dialogue and Tacit Knowledge for Knowledge Transfer' in Experimental Psychology, 1995, with the University of Cambridge, UK. She has been a Research Scientist with NTT's Communication Science Laboratories (CSL) and ATR (Kyoto) in Japan (1997-1999) and held a Joint position with CKIR, Finland and CSLI (Centre for the Study of Language and Information) Stanford University (2000-2003) and was a Senior Research Fellow at Middlesex University, London, UK (2004-2009). Her work has investigated the processes of transformation in tacit knowing in communication.

Abhishek Hazra is a visual artist based in Bangalore. His work explores the intersections between technology and culture through the narrative device of a 'visual fable'. He is interested in the social history of scientific practices, and his current, ongoing project attempts to explore the history of science research in colonial India. Hazra works with animated shorts and digital slideshows that often integrate textual fragments drawn from fictional scenarios. He is also interested in the way in which the languages of science journalism and information visualization participate in the complex dynamics of 'knowledge dissemination' and 'translation'.

P. Vigneswara Ilavarasan is presently Assistant Professor in the Department of Humanities and Social Sciences at Indian Institute of Technology Delhi. He obtained his $\mathrm{PhD}$ (Sociology) from the Indian Institute of Technology (IIT), Kanpur. In the last few years, Dr. Ilavarasan did extensive research on the culture of the IT industry, India's booming sector since the mid 1990s.

Rein Jansma is architect of many public buildings and mobility throughout the Netherlands, Dubai, Georgia and more. Around 1990, together with Moshe Zwarts, Rein Jansma founded Zwarts and Jansma architects. They build soccer stadiums, railway stations, bridges, tunnels and other mobility infrastructure. In 1992, Zwarts and Jansma created the Dutch Pavilion on the world expo in Seville.

Priya Kaul is psychologist and a practicing therapist in Bangalore, India. She is consultant with CWSolution, a firm that facilitates workshops on leadership development, cross-cultural awareness and professional skills. Kaul has over 10 years of experience with business development.

Bryony Lavery is a British dramatist, known for her successful and award-winning 1998 play Frozen. In addition to her work in theatre, she has also written for television and radio. She has written books including the biography Tallulah Bankhead and The Woman Writer's Handbook and taught playwriting at Birmingham 
University. Her plays have a feminist undertone in them, and she has even written plays (like More Light which has only one male speaking role) with almost entirely female casts. She has written more than twenty plays since 1976.

Gavin MacFadyen is investigative journalist and Visiting Professor at City University London and the Director of the Centre for Investigative Journalism, an international training charity. MacFaydyen has been a Senior producer and director of many World in Action, Channel 4 Dispatches, BBC's Fine Cut, 24 h, Panorama, The Money Programme, MultiCultural Birmingham, PBS Frontline programmes from 1970 to the present.

Geetha Narayanan has been at the forefront of the developing digital industry in Bangalore, India's renowned ICT centre for several decades now. Being the founder and director of the Srishti School of Art Design and Technology, she has been developing and implementing programs that blend design thinking, problem, project or place-based learning with new digital technologies. Geetha Narayanan is formally trained in mathematics and international education. Currently, she is finishing her dissertation 'Complexity and System Thinking, Memetics, New Technologies, Learning and Social Change' at Sheffield Hallam University.

Jogi Panghaal is concerned with the shift from product to service design, with a special focus on how craftsmanship and traditional artisan communities can be an inspiration for the design of services in current modern societies. For many years, Panghaal was a contributor to Doors of Perception, a conference and network in which design, industry and social science collaborate to develop new ideas for service design. Panghaal graduated in Product Design from the National Institute of Design (NID) in Ahmedabad, India in 1977. Mr. Panghaal has been a visiting teacher at National Institute of Design in India, ID, at Les Ateliers Paris, at the School of Planning and Architecture, Delhi and at the Gerrit Rietveld Academy in Amsterdam.

Vibodh Parthasarathi studies the music industry in the context of the Indian communication industry under globalization, comparative media policy, and environmental movements and communication practices. His association with the media industry in India and abroad has varied from being a consultant, television producer and documentary director. He is one of the series editors of the Sage series on 'Communication Processes'. Currently, Parthasarathi is reader at the Centre for Culture, Media and Governance at Jamia Millia Islamia in Delhi.

Thomas Quillinan is a Security Researcher at the D-CIS Research Lab in Delft, in the Netherlands. He received a $\mathrm{PhD}$, in the area of Security for Distributed Systems, and an MSc in Computer Science from University College Cork in Ireland.
Aditya Dev Sood is the Founder and CEO of the Center for Knowledge Societies in Bangalore and Delhi, where he directs design and innovation projects involving peer-topeer networks, interactive services and interface design, as well as product design. With foundational training in Architecture and Critical Theory from the University of Michigan, he is a former Fulbright Scholar with doctorates in Socio-Cultural Anthropology and Sanskrit Philology from the University of Chicago.

Sander van Splunter is a researcher at the Intelligent Interactive Distributed Systems (IIDS) (http://www.iids. org) at VU University, Amsterdam, and at D-CIS laboratory in Delft. He has a background in Artificial Intelligence. His central research topic is autonomous adaptation of complex systems.

Carol Upadhya is associate professor at the National Institute of Advanced Studies in Bangalore. She has widely published and also made three ethnographic films 'Coding Culture: Bangalore's Software Industry' in collaboration with Gautam Sonti (NIAS-IDPAD project 2006). With A R Vasavi, she edited the revealing study 'In an Outpost of the Global Economy: Work and Workers in India's Information Technology Industry' (New Delhi: Routledge, 2008).

Inge van der Vlies is professor of Constitutional Law and Art and Law at the University of Amsterdam. She has served as part-time judge to the higher court in Amsterdam. Her present research focuses on the relationship of art and laws. Within this, ambit issues like cultural diversity, cultural identity, freedom of expression and the character of ownership of cultural good are important. She has widely published on a variety of perspectives on legislation, including international developmental perspectives. She is member of the Board of the European Association for Legislation and editor of the Dutch Judicial Journal. Professor van der Vlies was also specialized in art and law. She is vice-chair of the Dutch commission on the Restitution of Artworks from World War II, is board member of the national broadcaster VPRO and is advisor to the board of the Stichting Democratie en Media, which is owner of some of the largest newspapers in the Netherlands. Van der Vlies is a highly respected counsellor and mediator in political and legislative issues. She chairs a variety of special commissions for local and national government agencies in the Netherlands. Van der Vlies also worked as a counsellor for the promotion of the rule in various countries like Indonesia, Ethiopia and former eastern European countries.

Martijn Warnier graduated with a Masters of Science in Cognitive Artificial Intelligence from Utrecht University, the Netherlands, in the beginning of 2002. He did his PhD in the Security of Systems group at the Radboud University in Nijmegen. His research focused on Language-based Security and the mathematical formalization of properties 
such as non-interference, confidentiality and integrity. For the last 3 years, he has worked as a Postdoctoral Researcher in the Intelligent Interactive Distributed Systems group at the VU University, Amsterdam. Since September 2009, he is appointed the position of assistant professor at the TU Delft. His current research interests, besides security, include the interdisciplinary research field of Computer Science and (Computer) Law, and Selforganizing and Autonomic systems. In his free time, Martijn Warnier loves acting in the theatre.

Rebekah Wilson obtained a degree in Composition at Victoria University of Wellington, New Zealand in 1996. Working as an independent composer, electronic musician, installation artist and filmmaker, Rebekah Wilson has performed and lectured in many venues and festivals. Earlier this century, she held the position of Artistic CoDirector at STEIM (Studio for Electronic and Instrumental Music), where she curated and participated in international festivals, workshops and education programmes for live electronic and instrumental music, installations and film. Since 2004, she is the co-founder and director of technology for an international software development company, Source-Elements, delivering highfidelity real-time audio delivery over IP. She is currently living between Barcelona and New Zealand and has taken up surfing.

\section{References}

Brazier FMT, van der Veer G (2009) Interactive distributed and networked autonomous systems: delegation or participation. In: Proceedings of the human interaction with intelligent and networked systems workshop (HINNS 2009), Florida
Brazier FMT, Oskamp A, Prins JEJ, Schellekens MHM, Wijngaards NJE (2004) Law-abiding and integrity on the internet: a case for agents. AI Law 12(1-2):5-37

Castells Manuel (1996) The rise of the networked society. Blackwell Publishing, Oxford

Castells M (2009) Communication power. Oxford University Press, Oxford

Damasio A (2004) Looking for spinoza, joy, sorrow and the feeling brain. Vintage, Random House, London

Fukuyama F (1995) Trust, social virtues and the creation of prosperity. Free Press Paperbacks, Simon Schuster, New York

Giddens A (1984) The constitution of society, outline of the theory of structuration. Polity Press, Cambridge

Hoven van den MJ (2005) Design for values and values for design information age+. J Aust Comp Soc 7(2):4-7

IJsselsteijn WA (2004) Presence in depth, PhD dissertation, Technische Universiteit Eindhoven

Kleiner A (2002) Karen Stephenson's quantum theory of trust. In strategy + business, fourth quarter. Retrieved from Web Site: http://www.strategy-business.com. Accessed 24 Nov 2006

Kuhn TS (2000) The road since structure: philosophical essays, 1970-1993, with an autobiographical interview. In: James C, John $\mathrm{H}$ (eds). The University of Chicago Press: Chicago. http://www.amazon.com/dp/0226457982/ref=rdr_ext_tmb

Lunenfeld P (2003) The design cluster, preface. In: Laurel B (ed) Design research: methods and perspectives. MIT press, Cambridge, pp 10-15

Nevejan C (2007) Presence and the design of Trust. PhD dissertation, University of Amsterdam

Nevejan C (2009) Witnessed Presence and the YUTPA framework. In PsychNology journal 'ethics in presence and social presence technology', vol 7 (1). www.psychnology.org

Nevejan C, Brazier F (2010) Witnessed Presence in merging realities in healthcare environments. In: advanced computational intelligence paradigms in healthcare 5 , studies in computational intelligence, 2011, vol 326/2011, Springer, pp 201-227

Oliver K (2001) Witnessing, beyond recognition. University of Minnesota Press, Mineapolis

Riva G, John AW, Eva LW (2004) The layers of presence: a biocultural approach to understanding presence in natural and mediated environments. CyberPsychol Behav 4(7):402-416

Tokoro M, Steels L (2003) The future of learning. IOS Press, Amsterdam 